\title{
An Optimized Model for Linear Feature Extraction from Satellite Image
}

\author{
Leila Mohammadnia and Jalal Amini
}

\begin{abstract}
This paper proposes an optimized mathematical model for linear feature extraction from satellite images. The model first uses the Ant Colony Optimization (ACO) to detect the edges from the satellite image. Next the line plausibility map is used as the external energy in a snake model to identify and extract the linear features like roads edges in image. Our approach is validated by a series of tests on satellite images.
\end{abstract}

Index Terms-Ant colony optimization, image edge detection, road, satellite image, snake, line plausibility map.

\section{INTRODUCTION}

In the last years, many approaches developed to deal with detecting linear features on satellite images [1]-[10]. Snakes, also called Active Contour Models, were introduced by Kass [11], and are used for extracting linear features in images. Compared with other extraction methods for linear features, the main advantage of snakes is that geometric properties of the features can be embedded constraints and used directly to guide the search in road extraction. This paper proposes an improved approach based on the ant colony optimization algorithm to detect the edges in the image and used as external forces in the snake model. In summary the innovation aspects insist in the ant colony optimization solution in the snake model.

At the rest of this paper, section 2 illustrates material and method. The experimental results will be given in section 3 and finally section 4 presents the conclusions and remarks.

\section{MATERIAL AND METHOD}

\section{A. Line Plausibility Map Extraction}

The edge information and line plausibility map in snake model is detected by Ant colony optimization (ACO) method. The aim of the Ant Colony optimization is iteratively find the best solution of the problem through a guided search over the solution space, by constructing the pheromone information. Suppose totally K ants are applied to find the best solution in a space $\chi$ that consists of $\mathrm{M} 1 \times$ M2 nodes. There are two basic issues in the ACO process; that is, the probabilistic transition matrix $p^{(n)}$ and the

Manuscript received May 18, 2012; revised June 12, 2012

L. Mohammadnia is with Science and Research Branch, Islamic Azad University .Tehran, Iran (e-mail: 1mohamadnia@gmail.com).

J. Amini is with Department of geomantic engineering, collage of Engineering, University of Tehran, and Tehran, Iran (e-mail: lmohamadnia@gmail.com). update of the pheromone matrix $\tau^{(n)}$. First, at the $n$-th construction-step of $A C O$, the $k$-th ant moves from the node $i$ to the node $j$ according to a probabilistic action rule, which is determined by [12]

$$
p_{i, j}^{n}=\frac{\left(\tau_{i, j}^{(n-1)}\right)^{\alpha}\left(\eta_{i, j}\right)^{\beta}}{\sum_{j \in \sum \Omega}\left(\tau_{i, j}^{(n-1)}\right)^{\alpha}\left(\eta_{i, j}\right)^{\beta}} \quad \text { if } j \in \Omega_{i}
$$

where $\tau_{i, j}^{n-1}$ is the pheromone information value of the arc linking the node $i$ to the node $j ; \Omega_{i}$ is the neighborhood nodes for the ant $a_{k}$ given that it is on the node $i$; the constants $\alpha$ and $\beta$ represent the influence of pheromone information and heuristic information, respectively; $\eta_{i, j}$ represents the heuristic information for going from node $i$ to node $\mathrm{j}$, which is fixed to be same for each construction-step. Second, the pheromone matrix needs to be updated twice during the ACO procedure. The first update is performed after the movement of each ant within each construction step. To be more specific, after the move of the k-th ant in the $n$-th construction-step, the pheromone matrix is updated as [12]

$$
\tau_{i, j}^{n-1}= \begin{cases}(1-\rho) \tau_{i, j}^{(n-1)}+\rho \Delta_{i, j}^{(k)} & \text { if }(i, j) \text { belongs to the tour } \\ \tau_{i, j}^{(n-1)} & \text { otherwise }\end{cases}
$$

where $\rho$ is the evaporation rate and $\Delta_{i, j}^{(k)}$ is determined by the heuristic matrix; that is, $\Delta_{i, j}^{(k)}=\eta_{i, j}$. The second update is performed after the move of all $\mathrm{K}$ ants within each construction-step; and the pheromone matrix is updated as [12]

$$
\tau^{(n)}=(1-\psi) \tau^{(n-1)}+\psi \tau^{(0)}
$$

where $\psi$ is the pheromone decay coefficient. There are two issues in the construction process. The first issue is determinating the heuristic information $\eta_{i, j}$ in (1). In this paper, it is proposed to be determined by the local statistics at the pixel position $(\mathrm{i}, \mathrm{j})$ as

$$
\eta_{i, j}=\left(I(i, j)-I_{m}(i, j)\right)^{2}
$$


where $I(i, j)$ is the intensity value of the pixel at the position $(i, j)$ of the image $\mathbf{I} . I_{m}(i, j)$ is the mean value of the intensity values of a window with size of $3 \times 3$ centered on $(i, j)$. The second issue is to determine the permissible range of the ant's movement in (1) at the position. In this paper, it is proposed to be either the 4-connectivity neighborhood or the 8-connectivity neighborhood, both of which are demonstrated in Fig. 1.
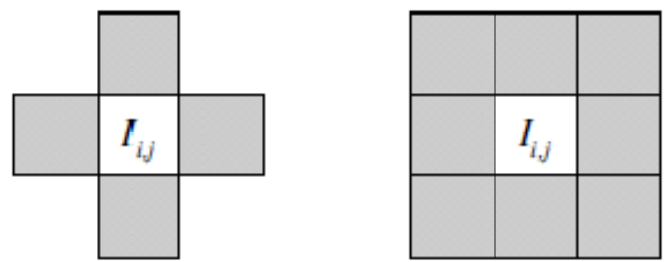

Fig. 1. The ants movement in (a) 4-connectiving and (b) 8-connectiving

In decision process a binary decision is made at each pixel location to determine whether it is edge or not, by applying threshold $\mathrm{T}$ on the final pheromone matrix $T^{(N)}$. In this paper, the above-mentioned $\mathrm{T}$ is proposed to be adaptively computed based on the method developed in [13]

\section{B. Snake-Ant Model}

The solution to be used is the snake approach .Snake is a parametric curve which is allowed to deform from some arbitrary initial location toward the desired final location by minimizing an energy function. In the continues domain, the snake is defined as a parametric curve, $r(s, t)=(x(s, t), y(s, t))$, Where $s$ is proportional to arc length, $t$ is the current time, and $x$ and $y$ are the curve's image coordinates. The snake minimizes an energy function based on internal and external constraints at time $t$.

$$
E_{t o t}(r(s, t))=\eta E_{\text {int }}(r(s, t))+E_{\text {ext }}(r(s, t))
$$

where: $E_{\text {int }}$ is the internal energy, depend $\mathrm{s}$ on the geometric characteristics of the roads such as length and curvature and $E_{\text {ext }}$ is the external energy depends on the plausibility that a line is passing through a pixel or not. To achieve the line plausibility map, edge detector from subsection 2.1 is chosen. $\eta$ is a regularization parameter. The energy function is built such that its global minimum coincides with the solution

$$
r_{0}=\min _{r} E_{t o t}(r(s, t))
$$

The equation (6) is usually implemented using finite elements [14] - [16]. This method offers an accurate discritization of the derivative, leading to greater accuracy. Discrediting the parametric curve $r(s, t)$, for $t=t_{0}$, into finite elements leads an expression for each elements, given by $r^{e}(s)=\langle\mathbf{N}(s)\rangle \cdot\left[\mathbf{V}^{e}\right]$ Where: $<\ldots>$ denotes row vectors and $[\ldots]$ matrices or column vectors. $\mathrm{N}(\mathrm{s})$ is a set of shape or basis functions $\left\langle\mathbf{N}_{1}(s), \mathbf{N}_{2}(s), \ldots, \mathbf{N}_{m}(s)\right\rangle$ defining the interpolating curves and $\left[\mathbf{V}^{e}\right]$ is a two column matrix, $\left[\mathbf{X}^{e}, \mathbf{Y}^{e}\right]$, of control points. So, as shown in Fig. 2, the curve (here road edge) $r(s)$ can be approximated using finite elements $r^{e}(s)$

$$
r(s)=\sum_{e=1}^{N} r^{e}(s)
$$

Thus, minimizing $E_{t o t}^{e}$ for each element leads to $\partial E_{t o t}^{e} / \partial V^{e}=0$, thus

$$
\left(\alpha \cdot\left[k_{1}\right]+\beta\left[k_{2}\right]\right) \cdot\left[\mathbf{V}^{e}\right]+\left[f^{e}\right]=\left[k^{e}\right] \cdot\left[\mathbf{V}^{e}\right]+\left[f^{e}\right]=0
$$

where:

$$
\left[K_{1}\right]=\int_{S}\left[\mathbf{N}_{s}\right] \cdot\left\langle\mathbf{N}_{s}\right\rangle d s \quad\left[K_{2}\right]=\int_{S}\left[\mathbf{N}_{s s}\right] \cdot\left\langle\mathbf{N}_{s s}\right\rangle d s
$$

$\left[f^{e}\right]$ is the external forces vector from subsection 2.1 applied on the control nodes of element $e$ of the snake.

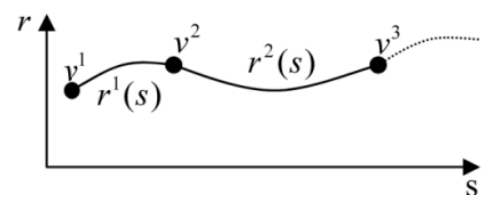

Fig. 2. Elements of a road

Thus, the snake -Ant model takes as input the road database or initial points coordinates and a satellite image the output is the extracted road edges according the illustration of this paper. The snake is stopped if the relative difference between two successive iterations becomes smaller than a fixed threshold.

\section{EXPERIMENTAL RESULTS}

In this section, an experimental with result will be given. Experimental is part of image from a city (kish) in Iran as Fig. 3(a), Fig. 3(b) shows the line plausibility map and Road edges are shows in Fig. 3 (c) after the snake-ant modeling.

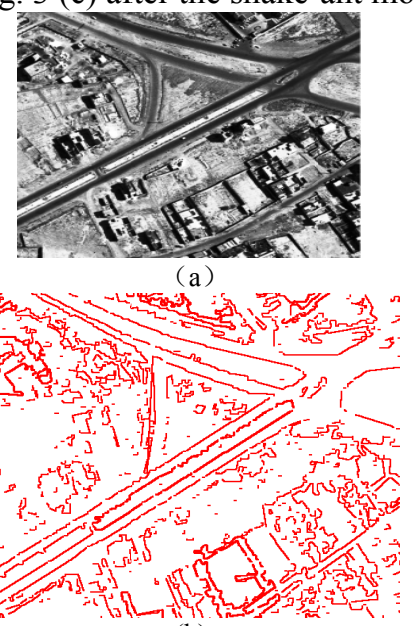

(b) 


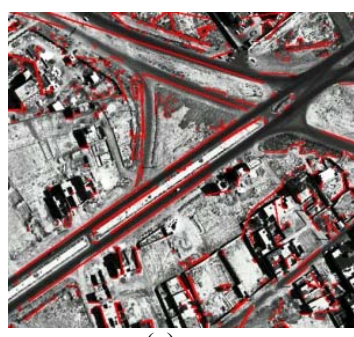

(c)

Fig. 3. (a) original image, (b) line plausibility map, (c) extracted road vector

For geometric accuracy assessment of the snake-Ant algorithm the road edges are extracted both manually as reference or ground-truth and automatically so that the accuracy of results could be assessed. To measure the impact of the restoration step, the snake localization process is achieved before and after restoration. Since the groundtruth is known, a quantification of the localization

Error is possible for both cases. Indeed, the obtained results are sampled and compared to the ground-truth vectors. This is achieved by measuring the average distance between the ground-truth and the obtained results as follows:

$$
\bar{D}=\frac{1}{N_{\text {sample }}} \sum_{i=1}^{N_{\text {ample }}} \sqrt{\left(x_{i}-x_{i}^{t}\right)^{2}+\left(y_{i}-y_{i}^{t}\right)^{2}}
$$

where $\left(x_{i}, y_{i}\right)$ the coordinates of the obtained solution using the snake-Ant, and $\left(x_{i}^{t}, y_{i}^{t}\right)$ are the coordinates of the ground-truth roads. Table I gives the obtained value for experimental.

TABLE I: AVERAge DistANCE BETWEEN THE OBTAINED SOLUTION AND THE GROUND - TRUTH

\begin{tabular}{|lll|}
\hline Experimental & $\bar{D}$ (Pixel) & 1.32 \\
\hline
\end{tabular}

The result showed that the proposed method is efficient in automatic linear feature extraction in satellite images.

Conclusions

This paper developed a snake-ant model, for identifying and extracting linear feature such as road edges from the satellite images. Ant colony optimization (ACO) was first introduced to detect the edges from the satellite images. The ACO-based edge detection approach was able to establish a pheromone matrix and line plausibility map that represents the edge information at each pixel position of the image.
Next the line plausibility map was used as the external energy in the snake model to extract the linear features like roads edges from images.

The accuracy of the extracted road vectors was evaluated both visually and quantitavely. From viewpoint in visually, the extracted roads was close to the roads on the original image. From viewpoint in quantitavely, the statistical results confirmed that the proposed approach is also efficient with average accuracy about 1.3 pixels.

\section{REFERENCES}

[1] F. Tupin, H. Maître, J. F. Mangin, J. M. Nicolas, and E. Pechersky, "Detection of linear features in SAR images: Application to road network extraction," IEEE Trans. Geosci. Remote Sensing, vol. 36, pp. 434-452, 1998.

[2] B. K. Jeon, J. J. Jang, and K. S. Hong, "Map-based road detection in space borne synthetic aperture radar images based on curvilinear structures extraction," Opt. Eng, vol. 39, no. 9, 2000.

[3] J. Chanussot, G. Mauris, and P. Lambert, "Fuzzy fusion techniques for linear features detection in multitemporal SAR images," IEEE Trans. Geosci. Remote Sensing, vol. 37, pp. 1292-1305, 1999.

[4] Y. Wang and Q. Zheng, "Recognition of roads and bridges in SAR images," Patt. Recognit, vol. 31, no. 7, pp. 965-977, 1998.

[5] M. F. A. Fortier, D. Ziou, C. Armenakis, and S. Wang, "Automated correction and updating of road databases from high-resolution imagery," Can. J. Remote Sens, vol. 27, no. 1, pp. 76-89, 2009.

[6] H. Mayer, I. Laptev, A. Baumgartner, and C. Steger, "Automatic road extraction based on multi-scale modeling, context and snakes," Int. Arch. Photogramm. Remote Sens, pt. 3-2W3, vol. 32, pp. 106-113, 1997.

[7] A. Baumgartner, C. T. Steger, H. Mayer, and W. Eckstein, "MultiResolution Semantic objects, and context for road extraction," in Semantic Modeling for the Acquisition of Topographic Information from Images and Maps, W. Fröstner and L. Plümer, Eds. Basel, Switzerland, pp. 140-156, 1997.

[8] N. Merlet and J. Zerubia, "Integration of Global Information for Roads Detection in Satellite Images," INRIA, 3239, 1997.

[9] C. Hivernat, X. Descombes, S. Randriamasy, and J. Zerubia, "Miseen Corespondance et Recalage de Graphes: Application aux Réseaux Routiers Extraits d'un Couple Carte/Image," INRIA, 3529, 1998.

[10] D. Klang, "Automatic detection of changes in road database using sattelite imagery," in Proc. Int. Archives Photogrammetry and Remote Sensing, vol. 32, pp. 293-298, 1998.

[11] M. Kass, A. Witkin, and D. Terzopoulos, "Snakes: Active contour models," Int. J. Comput. Vis, vol. 1, no. 4, 1988, pp. 321-331.

[12] M. Dorigo, M. Birattari, and T. Stutzle, "Ant colony optimization," IEEE Computational Intelligence Magazine, vol. 1, pp. 28-39, 2006.

[13] N. Otsu, "A threshold selection method from gray level histograms," IEEE Trans. Syst, Man, Cybern, vol. 9, pp. 62-66, 1979.

[14] M. Kisworo, S. Venkatesh, and G. A. W. West, "Detection of curved edges at subpixel accuracy using deformable models," IEE Proc. Inst. Elect. Eng, vol. 142, no. 5, pp. 304-312, 1995.

[15] J. A. Sethian, Level Set Methods and Fast Marching Methods: Evolving Interfaces in Computational Geometry, Fluid Mechanics, Computer Vision and Materials Science. Cambridge, U.K.: Cambridge Univ. Press, 1999.

[16] R. Deriche and O. Faugeras, "Tracking line segments," Image Vis. Comput., vol. 8, no. 4, pp. 261-270, 1990. 